\title{
The Excess Attenuation of Electric Field in the Presence of Deciduous Trees
}

\author{
Jovana G. Perovic and Dragan I. Olcan, Member, IEEE
}

\begin{abstract}
Excess attenuation of electric field of an electromagnetic wave propagating through deciduous forest is approximated with two novel analytical formulas. The coefficients of those formulas were obtained through a computationally intensive optimization process by fitting the simulation results obtained from stochastic 3D and 2D models of forests. The electric field was either vertically or horizontally polarized, at frequencies $30 \mathrm{MHz}, 60 \mathrm{MHz}$ and $100 \mathrm{MHz}$. According to numerical experiments, these formulas predicted the excess attenuation within several decibels, up to at least $100 \mathrm{MHz}$. Nevertheless, the proposed formulas matched very well the measured data from literature below $1 \mathrm{GHz}$, and neatly followed the measurements even up to $10 \mathrm{GHz}$. Therefore, the frequency range considered in the numerical experiments can be extrapolated at least tenfold.
\end{abstract}

Key words - Attenuation, approximation formula, electromagnetic waves, modeling, full-wave electromagnetic simulation, trees.

\section{INTRODUCTION}

$\mathrm{I}_{\mathrm{p}}^{\mathrm{N}}$ $\mathrm{N}$ free space, the intensity of Poynting vector is proportional to $r^{-2}$, where $r$ is the distance traveled by an electromagnetic (EM) wave, radiated from a transmitting antenna [1]. In addition, if obstacles like trees, buildings, people, etc., are present along the propagation path, the EM field is typically attenuated more than in free space. Radiowave propagation in the presence of vegetation is of interest for many practical applications like radio coverage planning, ad hoc radio communications, military communications, etc. Some numerical models that predict the excess attenuation in forests were developed [2]-[9] and measurements for different types of vegetation were carried out [4], [10]-[12]. The attenuation in urban environments with vegetation was studied in [13] and [14].

Paper received April 27, 2017; revised July 10, 2017; accepted July 13, 2017. Date of publication July 31, 2017. The associate editor coordinating the review of this manuscript and approving it for publication was Prof. Branko Kolundžija.

This paper is a revised and expanded version of the paper presented at the 24th Telecommunications Forum TELFOR 2016 [15].

The work presented here is partially sponsored through the project TR32005 of the Serbian Ministry of education, science and technological development.

Jovana G. Perovic (contact author), School of Electrical Engineering, University of Belgrade, Bulevar kralja Aleksandra 73, 11120 Belgrade, Serbia (phone: 381-11-3218386, e-mail: jovanap@etf.rs ).

Dragan I. Olcan, School of Electrical Engineering, University of Belgrade, Bulevar kralja Aleksandra 73, 11120 Belgrade, Serbia (phone: 381-11-3218329, e-mail: olcan@etf.rs ).
The work presented here is an extension of [15].

Approximation formulas for the excess attenuation due to the presence of vegetation are given in International Telecommunication Union (ITU) recommendation [14].

The excess attenuation at a terrestrial path with the receiver in a forest and the transmitter outside the forest is approximated with:

$$
A_{\mathrm{ev}}=A_{\mathrm{m}}\left(1-\exp \left(-d \gamma / A_{\mathrm{m}}\right)\right) \text {, }
$$

where $A_{\mathrm{m}}[\mathrm{dB}]$ and $\gamma[\mathrm{dB} / \mathrm{m}]$ are empirically found parameters (influenced by the forest), and $d[\mathrm{~m}]$ is the total length of the radio path through the forest [14].

For communications that use satellite links, the excess attenuation is approximated with:

$$
A_{\mathrm{ev}}=A f^{B} d^{C}(\theta+E)^{G},
$$

where $f[\mathrm{MHz}]$ is the operating frequency, $d[\mathrm{~m}]$ is the total length of the radio path through the forest, $\theta$ [degrees] is the elevation angle and $A, B, C, E$ and $G$ are empirically found parameters [14].

We show that (1) and (2) can be combined together in a single analytical formula having in mind that (1) works for electrically shorter distances (i.e., communication at lower frequencies), while (2) works for electrically longer distances (i.e., communications at higher frequencies).

The paper is organized as follows. In section II we briefly outlined stochastic 2D and 3D modeling of trees. In section III we explained the calculation of the excess attenuation. In section IV we outlined the proposed approximation formula. The fitted parameters of that formula were listed, as well. In section $\mathrm{V}$ we presented results from numerical experiments that were used for verification of the formula. To compare our analytical estimations of the excess attenuation with measured results from the literature, we presented results for per-unit-length excess attenuation that fit measured data in section VI. Finally, in section VII we concluded the paper.

\section{ELECTROMAGNETIC MODELING OF TREES}

In order to numerically calculate the excess attenuation of EM wave propagating within deciduous forests, we used both 2D models (i.e., cross-section) and 3D models of trees.

For 2D modeling, where one dimension (in this work it was $z$-coordinate of the global Cartesian coordinate system) was considered infinite, the trees were presented as dielectric circles with relative permittivity $\varepsilon_{\mathrm{r}}=10$ [7]. Simulations were performed using WIPL-D 2D solver. A code for automatic generation of stochastically distributed cross-sections and their sizes was implemented in MATLAB [16]. The radius of a circle (i.e., the cross-section of a tree) was a random variable with the uniform 
distribution between $0.25 \mathrm{~m}$ and $0.35 \mathrm{~m}$. Also, $x$ - and $y$-coordinates of the circle center were random variables with the uniform distribution. The input data for the generator were: the total number of trees $N$, dimensions of a (flat) rectangular surface where trees were distributed and the total number of models $M$. Each set of a forest cross section was written as one file and formatted as needed for WIPL-D 2D solver.

For 3D modeling we used in-house developed software for generating stochastic wire-based models of trees [6]. Those models were simulated using software WIPL-D Pro. Tree trunks and branches were modeled as conductive wires with distributed loadings. Again, positions of trees, radii of trunks, dimensions of branches, and their directions in 3D space were random variables with the uniform distribution. A typical tree was approximately $7.5 \mathrm{~m}$ high, with the average height of first braches of $4 \mathrm{~m}$.

In both $2 \mathrm{D}$ and $3 \mathrm{D}$ simulations, the excitation was a uniform time-harmonic plane wave, either vertically or horizontally polarized. Fig. 1a shows an example of a 3D model of forest illuminated with a vertically polarized EM wave, and Fig. $1 \mathrm{~b}$ depicts an example of 2D model illuminated with a horizontally polarized wave.

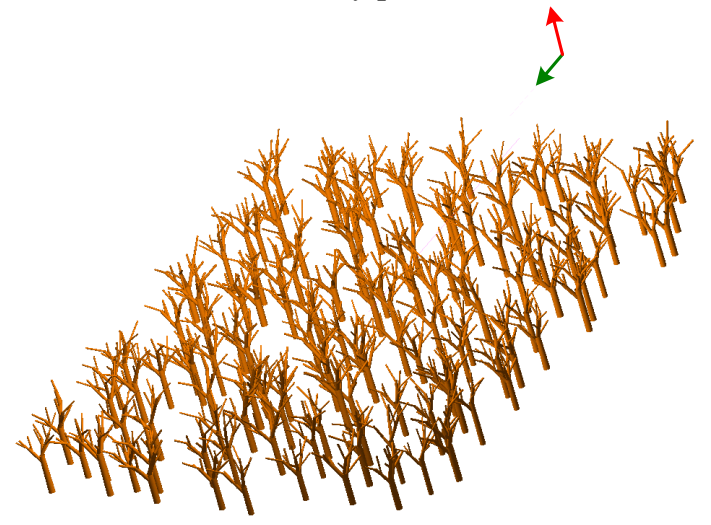

(a)

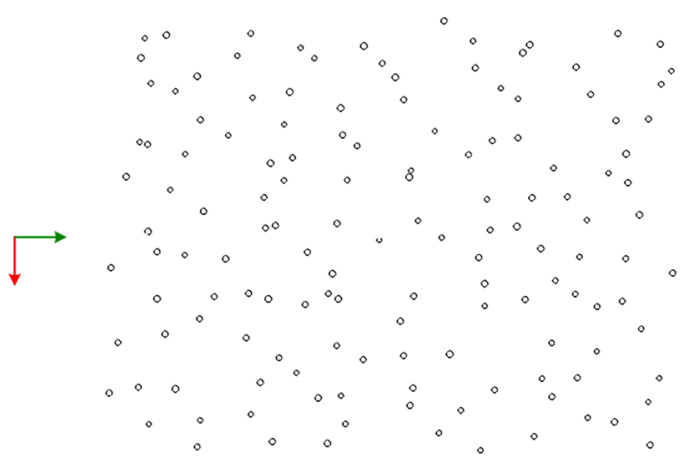

(b)

Fig. 1 (a) 3D model and (b) 2D model of a forest that consists of 140 random trees.

For the simulation we considered forests that consist of $20,40,70$ and 140 trees positioned on a rectangular surface of dimensions $45 \mathrm{~m} \times 60 \mathrm{~m}$. The surface density of trees normalized to one tree per square meter, $\rho$, was 0.0074 , $0.0148,0.0259$ and 0.0518 , respectively.

Both used solvers are based on the method of moments (MoM), which transforms an EM problem into solving a system of linear equations. The total number of the system matrix elements is $K^{2}$, where $K$ is the total number of unknown coefficients. For example, one 3D wire model with 140 trees at $100 \mathrm{MHz}$ had about 7,200 unknown coefficients for current approximation along wires, while one 2D dielectric model of 140 trees at $100 \mathrm{MHz}$ had about 6,700 unknown coefficients for the approximation of surface currents.

The impact of trees on a vertically polarized wave was correctly predicted using 3D wire models because there was only vertical (axial) current on wires in the model. However, those models couldn't be used for horizontally polarized excitation since the EM interaction between the tree trunk and the horizontally polarized EM wave did not exist in the model. Therefore, the attenuation for a horizontally polarized wave was considered only in 2D case, while the attenuation for a vertically polarized wave was observed from all models. Although the illuminating wave had only one (space) component of electric filed, in 3D models there were all three components of EM field in the forest, i.e., the 3D numerical EM analysis showed the change of the electric field polarization. This is usually called de-polarization in the literature [14].

\section{CAlCulating the EXCESS ATtenUATION}

The operating frequencies at which simulations were performed are $30 \mathrm{MHz}, 60 \mathrm{MHz}$ and $100 \mathrm{MHz}$.

The incident wave was either vertically or horizontally polarized, while the root-mean-square (rms) value of electric field was $E_{\text {inc }}=1 \mathrm{~V} / \mathrm{m}$. Note that a linear combination of vertical and horizontal polarization can yield an arbitrary polarized wave. Therefore, using results for these two distinct cases, we can predict the excess attenuation for arbitrary polarization of the excitation.

The ground below the trees was modeled as a perfect electric conductor (PEC), in the case of 3D models with vertical polarization which was a worst-case scenario.

Using numerical EM solvers we calculated the total (near) field at points that were $1.8 \mathrm{~m}$ above the ground, since we assumed that this height was a typical position for a hand-held receiver (i.e., near the head of the user). Additional numerical experiments yielded similar excess attenuation for all heights from $1.5 \mathrm{~m}$ to $2 \mathrm{~m}$ above the ground. The points where EM field was calculated were uniformly distributed on a flat rectangular surface of the width equal to one wavelength, $\lambda$, at the operating frequency, and of the length equal to the total length of the forest, as it is shown in Fig. 2. The distance between two neighboring points in both dimensions was not longer than $\lambda / 10$, so that we had sufficiently dense spatial sampling of EM field.

At one point in a forest, the excess attenuation of the electric field, in all considered cases, was calculated using:

$$
A_{\mathrm{ev}}[\mathrm{dB}]=20 \log _{10}\left(1 \frac{\mathrm{V}}{\mathrm{m}} / 1 \frac{\mu \mathrm{V}}{\mathrm{m}}\right)-20 \log _{10}\left(E / 1 \frac{\mu \mathrm{V}}{\mathrm{m}}\right)
$$

where $E$ was the rms of the electric field at the observation point.

We assumed that the incident wave traveled in the direction of $x$-axis (Fig. 2). The smallest $x$-coordinate in which we calculated the excess attenuation was designated as $d_{0}$ and attenuations at $N$ points with the same $x$-coordinate (but different $y$-coordinates) were designated 
as $A_{\mathrm{ev}}\left(d_{0}, y_{1}\right), A_{\mathrm{ev}}\left(d_{0}, y_{2}\right), \ldots, A_{\mathrm{ev}}\left(d_{0}, y_{N}\right)$, respectively. The maximum attenuation at the distance $d_{0}$ was calculated as:

$$
A_{\mathrm{ev}}\left(d_{0}\right)=\max \left\{A_{\mathrm{ev}}\left(d_{0}, y_{1}\right), \ldots, A_{\mathrm{ev}}\left(d_{0}, y_{N}\right)\right\} \text {. }
$$

At successive distance $d_{1}\left(d_{1}>d_{0}\right)$ the excess attenuation was calculated using:

$$
A_{\mathrm{ev}}\left(d_{1}\right)=\max \left\{A_{\mathrm{ev}}\left(d_{1}, y_{1}\right), \ldots, A_{\mathrm{ev}}\left(d_{1}, y_{N}\right), A_{\mathrm{ev}}\left(d_{0}\right)\right\} .
$$

This approach was chosen since it yielded monotonically nondecreasing excess attenuation as a function of distance, which was implicitly assumed in ITU recommendations [14]. Note that this approach yields a conservative estimation for the excess attenuation and is useful for practical applications when worst-case scenario is needed.

We generated 100 stochastic models for one polarization (either vertical or horizontal), one operating frequency and for the given total number of trees at the surface. The total number of stochastic models was chosen to be 100 because it yielded an $1 \mathrm{~dB}$ confidence interval for the mean-value of the excess attenuation with the probability of 0.95 . The mean value of the excess attenuation was calculated as:

$$
\overline{A_{\mathrm{ev}}}(d)=\frac{1}{100} \sum_{i=1}^{100} A_{\mathrm{ev} i}(d),
$$

where $A_{\mathrm{ev} i}(d)$ stands for the excess attenuation at distance $d$ calculated for the $i^{\text {th }}$ stochastic model using (4) and (5). With 100 models, the confidence interval with probability of 0.95 was calculated using [17]:

$$
\left[\overline{A_{\mathrm{ev}}}(d)-\frac{1.984 s(d)}{\sqrt{101}}, \overline{A_{\mathrm{ev}}}(d)+\frac{1.984 s(d)}{\sqrt{101}}\right],
$$

where $s$ was the estimation of the standard deviation calculated as:

$$
s(d)=\sqrt{\frac{\sum_{i=1}^{100}\left(A_{\mathrm{ev} i}(d)-\overline{A_{\mathrm{ev}}}(d)\right)^{2}}{100-1}} .
$$

\section{FORMULAS FOR ESTIMATION OF THE EXCESS ATTENUATION IN DECIDUOUS FORESTS}

We approximated the excess attenuations of the electric field using two different formulas.

The first considered formula for the approximation of the excess attenuation was:

$$
\overline{A_{\mathrm{ev}}}=B\left(1-\mathrm{e}^{-d \gamma}\right)+C d^{D} \frac{\frac{\pi}{2}+\tan ^{-1}(F d-E)}{\pi},
$$

where $B[\mathrm{~dB}], C[\mathrm{~dB} / \mathrm{m}], D, E, F[1 / \mathrm{m}]$ and $\gamma[1 / \mathrm{m}]$ were unknown parameters and $d[\mathrm{~m}]$ the transverse path through a woodland. The first addend follows (1) and it is dominant up to several wavelengths into the woodlands, while the second addend is from (2) and is dominant for longer distances $d$. The second addend includes multiplier $\frac{\pi / 2+\tan ^{-1}(F d-E)}{\pi}$ which provides a smooth transition between the addends, leading to a smooth function for the approximated excess attenuation. If $F d-E>\pi$, then $\frac{\pi / 2+\tan ^{-1}(F d-E)}{\pi}>0.9$ and consequently the second addend is dominant. This formula works for a single frequency.
The second considered formula was:

$$
\overline{A_{\mathrm{ev}}}=\left(B_{0} f^{m_{\mathrm{B}}}+C_{0} \rho^{n_{\mathrm{C}}}\left(\frac{d}{\lambda}\right)^{D}\right)\left(1-\exp \left(-\frac{d}{\lambda} \gamma_{0} \rho\right)\right),
$$

where the operating frequency, $f$, was given in $\mathrm{MHz}, \rho$ was the surface density of trees normalized to one tree per square meter, and the distance traveled by the wave within the woodland, $d$, was normalized to the operating wavelength $\lambda$. This formula covers a range of frequencies. Note that in [18] we used a similar formula for approximation of the excess attenuation within human crowds, as well.

Unknown (empirical) parameters in (9) and (10) were found using a nonlinear optimization in order to fit the approximation as close as possible to the calculated attenuation. To that aim, the cost-function for the optimization was defined as:

$$
\delta=\sqrt{\frac{1}{N} \sum_{k=1}^{N}\left(x_{\mathrm{s} k}-x_{\mathrm{a} k}\right)^{2}},
$$

where $N$ was the total number of points at which we calculated the attenuation, $x_{\mathrm{s} k}$ and $x_{\mathrm{a} k}$ were attenuations at the $k^{\text {th }}$ distance obtained from simulations and from the approximation formula, respectively. By finding the minimum $\delta$ we found the best fit for the calculated excess attenuation using (9) or (10).

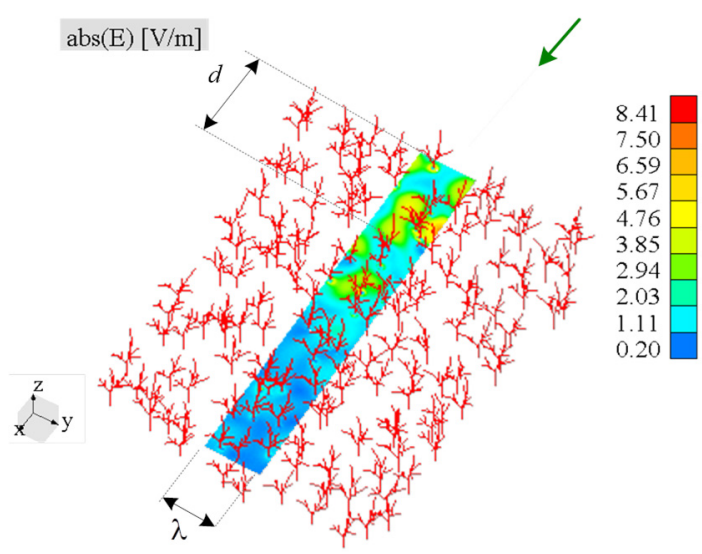

Fig. 2 An example of calculated (near) electric field.

For the optimization we used a repeated two-stage combination of random search and Nelder-Mead simplex algorithm [19]. It started with a random search using a number of cost-function evaluations (typically 100 to 500). The best-found solution was the starting point for the simplex algorithm. Finally, this two-stage approach was repeated for 10 to 50 times in order to increase the probability of finding the best possible approximation (i.e., the global optimum) [20].

At first, we optimized parameters for each attenuation setup using (9), and noticed the following. Parameters $B, C$ and $\gamma$ depend on the surface density of trees. Therefore, we included the normalized tree density, $\rho$, within those parameters as $B=B_{0} \rho^{n_{\mathrm{B}}}, C=C_{0} \rho^{n_{\mathrm{C}}}$ and $\gamma=\gamma_{0} \rho^{n_{\gamma}}$. Further, the parameter $D$ depends on the frequency and does not depend on tree density. Finally, parameters $E$ and $F$ have practically constant values around 0.8 and -0.8 , respectively. So, optimized parameters for all attenuation setups (for one polarization, one frequency and four 
different tree densities) were $B_{0}, n_{\mathrm{B}}, C_{0}, n_{\mathrm{C}}, D, \gamma_{0}$ and $n_{\gamma}$. The results are presented in Tables 1-4, where frequency $f$ is given in $\mathrm{MHz}$, parameter $B_{0}$ in $\mathrm{dB}$ and $C_{0}$ in $\mathrm{dB} / \mathrm{m}$, while all other parameters are dimensionless.

TABLE 1: PARAMETERS FOR VERTICAL POLARIZATION, 2D MODELS

\begin{tabular}{ccccccccc}
\hline \hline$f$ & $B_{0}$ & $n_{\mathrm{B}}$ & $C_{0}$ & $n_{\mathrm{C}}$ & $D$ & $\gamma_{0}$ & $n_{\gamma}$ & $\delta$ \\
\hline 30 & 20.5 & 0.01 & 23.1 & 0.32 & 0.30 & 677 & 2.26 & 1.78 \\
60 & 20.3 & 0.03 & 15.7 & 0.03 & 0.25 & 35.9 & 35.9 & 1.05 \\
100 & 16.2 & 0.00 & 23.4 & 0.03 & 0.16 & 3248 & 3248 & 1.1 \\
\hline \hline
\end{tabular}

TABLE 2: PARAMETERS FOR HORIZONTAL POLARIZATION, 2D MODELS

\begin{tabular}{ccccccccc}
\hline \hline$f$ & $B_{0}$ & $n_{\mathrm{B}}$ & $C_{0}$ & $n_{\mathrm{C}}$ & $D$ & $\gamma_{0}$ & $n_{\gamma}$ & $\delta$ \\
\hline 30 & 6.06 & 0.55 & 0.24 & 0.05 & 0.30 & 7.17 & 0.72 & 0.03 \\
60 & 13.8 & 0.74 & 4.24 & 0.44 & 0.25 & 9.99 & 0.14 & 0.06 \\
100 & 21.0 & 0.00 & 4.85 & 0.27 & 0.16 & 1.26 & 1.05 & 0.69 \\
\hline \hline
\end{tabular}

TABLE 3: PARAMETERS FOR VERTICAL POLARIZATION, 3D MODELS, ONE RECEIVED FIELD COMPONENT

\begin{tabular}{ccccccccc}
\hline \hline$f$ & $B_{0}$ & $n_{\mathrm{B}}$ & $C_{0}$ & $n_{\mathrm{C}}$ & $D$ & $\gamma_{0}$ & $n_{\gamma}$ & $\delta$ \\
\hline 30 & 8.50 & 0.00 & 17.6 & 0.08 & 0.30 & 0.36 & 0.28 & 1.82 \\
60 & 23.4 & 0.00 & 18.2 & 0.14 & 0.25 & 23.3 & 1.28 & 2.37 \\
100 & 24.4 & 0.01 & 19.4 & 0.05 & 0.16 & 1341 & 2.12 & 1.83 \\
\hline \hline
\end{tabular}

TABLE 4: PARAMETERS FOR VERTICAL POLARIZATION, 3D MODELS, ALL THREE RECEIVED FIELD COMPONENTS

\begin{tabular}{ccccccccc}
\hline \hline$f$ & $B_{0}$ & $n_{\mathrm{B}}$ & $C_{0}$ & $n_{\mathrm{C}}$ & $D$ & $\gamma_{0}$ & $n_{\gamma}$ & $\delta$ \\
\hline 30 & 1.27 & 3.00 & 4.66 & 7.20 & 0.30 & 1.03 & 0.14 & 1.63 \\
60 & 11.0 & 0.18 & 6.94 & 0.01 & 0.25 & 0.01 & 0.00 & 1.01 \\
100 & 1.94 & 0.00 & 11.1 & 0.00 & 0.16 & 0.01 & 0.00 & 0.98 \\
\hline \hline
\end{tabular}

Fig. 3 shows a typical curve of the excess attenuation. The presented one is for a vertically polarized EM wave for 2D case with 70 trees distributed on a rectangular surface of dimensions $45 \mathrm{~m} \times 60 \mathrm{~m}$. The solid line stands for attenuation, while the transparent shadow of the same color represents a $95 \%$ confidence interval, and the dotted line is the approximation curve, following (9). (The same presentation pattern is used throughout the whole paper.) The approximation is within the confidence interval (transparent blue), as it is shown in Fig. 3. Note the discrepancy between coefficients in Tables 3 and 4 which is the consequence of a significant change of field polarization within the woodlands.

Unlike (9), frequency dependence is included in (10). Therefore, except in two cases (2D models for horizontal polarization and $3 \mathrm{D}$ models for vertical polarization for all space components), optimization was done for all curves of one polarization, three frequencies and three densities. The exceptions were made in the cases when the optimization couldn't find acceptably good approximation. Results are presented in Tables 5-8, where frequency $f$ was given in $\mathrm{MHz}$, parameters $B_{0}$ and $C_{0}$ in $\mathrm{dB}$, while parameters $m_{\mathrm{B}}, n_{\mathrm{C}}$, $D$ and $\gamma_{0}$ were dimensionless.

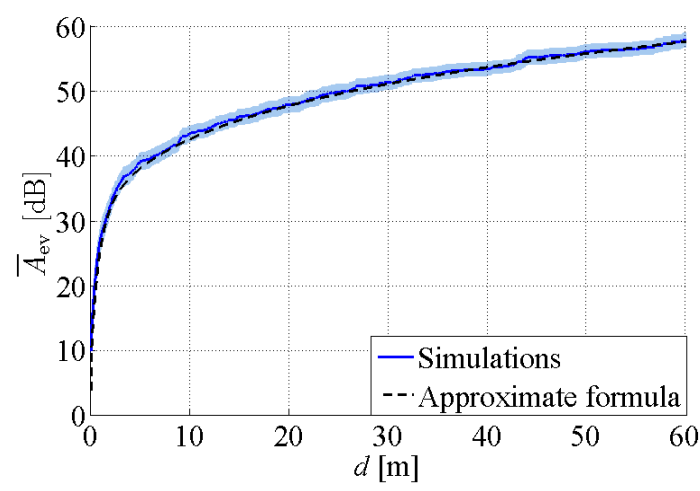

Fig. 3 Typical attenuation obtained from simulation (solid line) and the approximation (9) (dotted line).

TABLE 5: PARAMETERS FOR VERTICAL POLARIZATION, 2D MODELS

\begin{tabular}{cccccccc}
\hline \hline$f$ & $B_{0}$ & $m_{\mathrm{B}}$ & $C_{0}$ & $n_{\mathrm{C}}$ & $D$ & $\gamma_{0}$ & $\delta$ \\
\hline 30 & & & & & & & \\
60 & 3.421 & 0.297 & 31.89 & 0.067 & 0.211 & 179.9 & 3.65 \\
100 & & & & & & & \\
\hline \hline
\end{tabular}

TABLE 6: PARAMETERS FOR HORIZONTAL POLARIZATION, 2D MODELS

\begin{tabular}{cccccccc}
\hline \hline$f$ & $B_{0}$ & $m_{\mathrm{B}}$ & $C_{0}$ & $n_{\mathrm{C}}$ & $D$ & $\gamma_{0}$ & $\delta$ \\
\hline $\begin{array}{c}30 \\
60\end{array}$ & 0.003 & 1.614 & 31.81 & 1.384 & 0.635 & 192.9 & 0.29 \\
\hline 100 & 0.002 & 0.051 & 22.93 & 0.437 & 0.487 & 192.9 & 1.10 \\
\hline \hline
\end{tabular}

TABLE 7: PARAMETERS FOR VERTICAL POLARIZATION, 3D MODELS, ONE RECEIVED FIELD COMPONENT

\begin{tabular}{cccccccc}
\hline \hline$f$ & $B_{0}$ & $m_{\mathrm{B}}$ & $C_{0}$ & $n_{\mathrm{C}}$ & $D$ & $\gamma_{0}$ & $\delta$ \\
\hline 30 & & & & & & & \\
60 & 0 & 0.096 & 41.93 & 0.034 & 0.158 & 166.9 & 2.74 \\
100 & & & & & & & \\
\hline \hline
\end{tabular}

TABLE 8: PARAMETERS FOR VERTICAL POLARIZATION, 3D MODELS, ALL THREE RECEIVED COMPONENTS

\begin{tabular}{cccccccc}
\hline \hline$f$ & $B_{0}$ & $m_{\mathrm{B}}$ & $C_{0}$ & $n_{\mathrm{C}}$ & $D$ & $\gamma_{0}$ & $\delta$ \\
\hline 30 & 0.631 & 0.344 & 6.917 & 0 & 0.522 & 192.9 & 1.61 \\
\hline $\begin{array}{c}60 \\
100\end{array}$ & 7.533 & 0.057 & 2.598 & 0.008 & 0.559 & 192.9 & 1.36 \\
\hline \hline
\end{tabular}

Fig. 4 shows the excess attenuation of vertically polarized wave at $30 \mathrm{MHz}, 60 \mathrm{MHz}$ and $100 \mathrm{MHz}$, obtained from $2 \mathrm{D}$ models of $N$ trees distributed on a rectangular surface of dimensions $45 \mathrm{~m} \times 60 \mathrm{~m}$. Note that the same coefficients were used for all nine curves.

Even though from Figs. 3 and 4 can be seen that (9) gave better approximation, the advantage of (10) was its dependence of frequency.

\section{VERIFICATION OF ANALYTICAL ESTIMATIONS}

In order to further verify the formulas with optimized parameters, we made additional 20 highly complex models with 1600 stochastically positioned trees on a rectangular surface $200 \mathrm{~m} \times 200 \mathrm{~m}$, shown in Fig. 5. The excitation was a vertically polarized wave at $30 \mathrm{MHz}$. One model had 
about 50,000 unknowns, and it took about $2 \mathrm{hrs}$ to finish one such EM simulation on the best desktop computer we had access to. Hence, this numerical experiment took about two days.

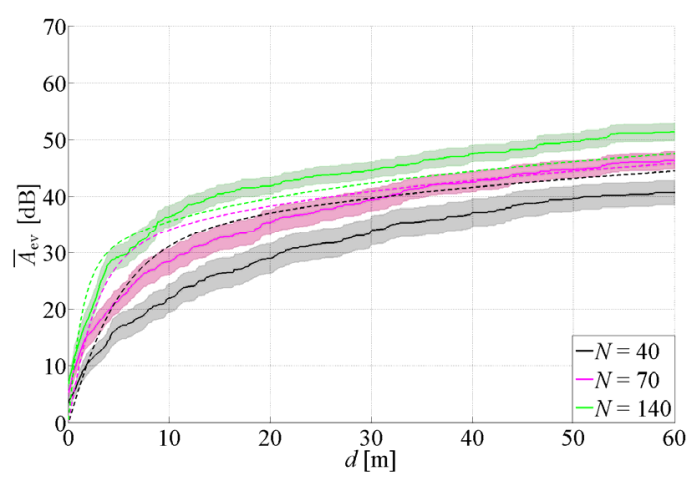

(a) $30 \mathrm{MHz}$.

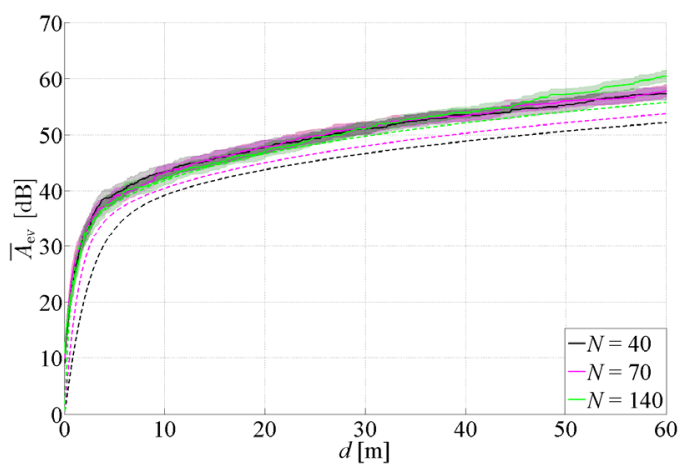

(b) $60 \mathrm{MHz}$.

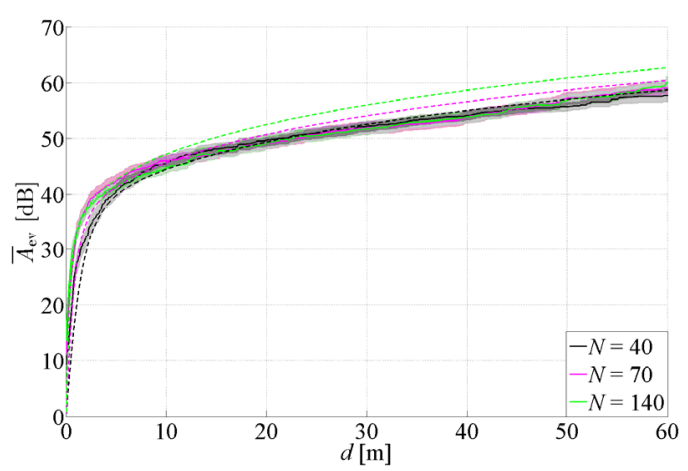

(c) $100 \mathrm{MHz}$.

Fig. 4 Typical attenuation obtained from simulation (solid line) and the approximation (10) (dotted line).

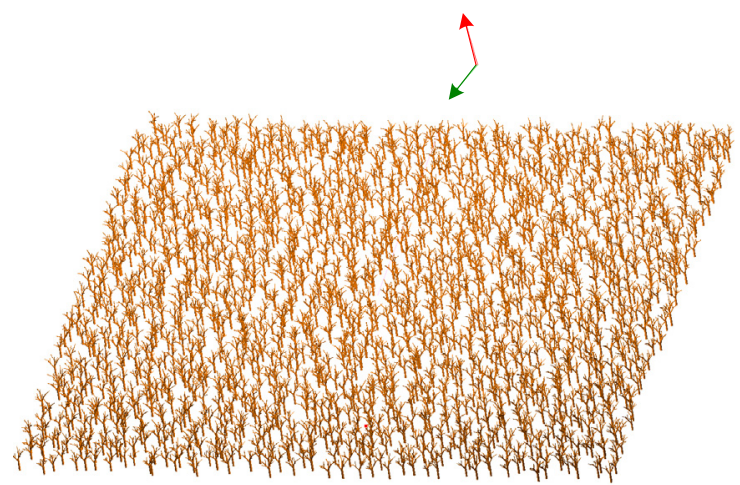

Fig. 5 A model of 1600 stochastically positioned trees.

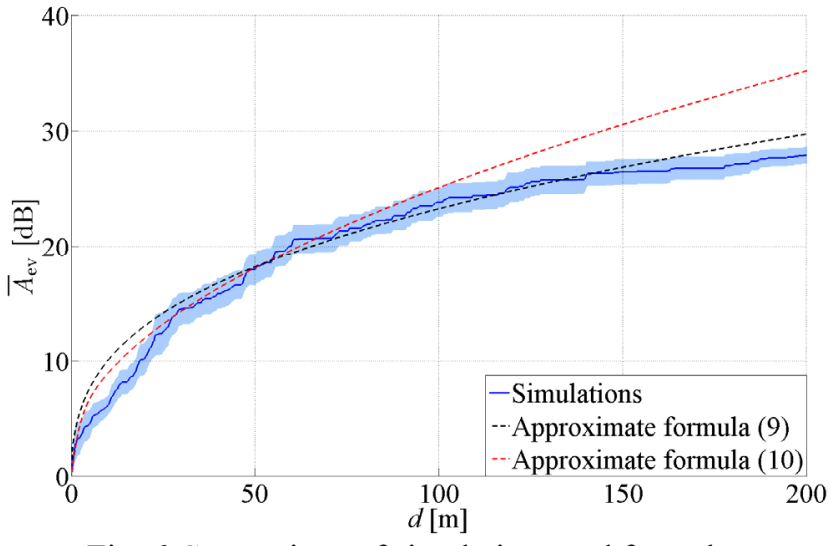

Fig. 6 Comparison of simulations and formulas.

The used parameters for (9) and (10) are given in the first row of Tables 4 and 8 . The results are shown in Fig. 6. It is observed that 20 wavelengths into the woodlands (9) yield the estimation for the excess attenuation with the average deviation of about $1.5 \mathrm{~dB}$. On the other hand (10) approximates the access attenuation with the average deviation of about $3.5 \mathrm{~dB}$. However, up to 10 wavelengths into the woodland, both formulas are similar, while at longer distances (9) gives a better prediction.

\section{COMPARISON TO MEASURED DATA}

Collected measured data from various experiments [21] are shown in Fig. 7. The attenuation is given in $\mathrm{dB} / \mathrm{m}$ as a function of frequency in the range from $100 \mathrm{~Hz}$ to $10 \mathrm{GHz}$. We compared these results to the excess attenuation obtained from proposed analytical formulas.

Measured data, namely Fig. 6 in [21], are mixed for both polarizations. So, we compared one arbitrary set of our EM models to the measured data, although the other sets yielded similar final results. We chose 2D models with horizontal polarization approximated with (10) as it covers a frequency range. First, we had to compute attenuation per meter from (10), as results in [21] are in that format.

To find the attenuation in $\mathrm{dB} / \mathrm{m}$ as a function of frequency, we used two approaches.

In the first approach, the attenuation in $\mathrm{dB} / \mathrm{m}$ was calculated using:

$$
\overline{A_{\mathrm{ev}}}=B_{0} f^{m_{\mathrm{B}}}\left(1-\exp \left(-\frac{d}{\lambda} \gamma_{0} \rho\right)\right) / d
$$

at distance $d=40 \mathrm{~m}$ in the frequency range from $100 \mathrm{MHz}$ to $10 \mathrm{GHz}$. Note that in (11) the second addend in the first parentheses from (10) is omitted, as its main contribution is to approximate the excess attenuation at distances up to several wavelengths. Using parameters from the first row of Table 6, we got a curve presented in green in Fig. 7. We observed that, for all considered frequencies, it estimates higher attenuation than measured. That is the consequence of the worst-case scenario that we assumed in (4) and (5), while in [21] the average excess attenuation was considered. For that reason, we computed the mean value for the attenuation, from our simulations. Namely, at one distance $d_{i}$ (in one stochastic model), the (mean) excess attenuation was:

$$
A_{\mathrm{ev}}\left(d_{i}\right)=\max \left\{\frac{1}{N} \sum_{k=1}^{N} A_{\mathrm{ev}}\left(d_{i}, y_{k}\right), A_{\mathrm{ev}}\left(d_{i-1}\right)\right\} .
$$


The first element, $\frac{1}{N} \sum_{k=1}^{N} A_{\mathrm{ev}}\left(d_{i}, y_{k}\right)$, is the mean value of the excess attenuation at distance $d_{i}$ and the second element $A_{\mathrm{ev}}\left(d_{i-1}\right)$ is the excess attenuation at previous distance, $d_{i-1}$. Note that both (5) and (12) yield a monotonically nondecreasing function of distance.

The optimizations were again used for finding parameters of (10) that yield the best fit. A solution for frequencies $30 \mathrm{MHz}$ and $60 \mathrm{MHz}$ is presented in Table 9.

TABLE 9: HORIZONTAL POLARIZATION 2D MODELS

\begin{tabular}{cccccccc}
\hline \hline$f$ & $B_{0}$ & $m_{\mathrm{B}}$ & $C_{0}$ & $n_{\mathrm{C}}$ & $D$ & $\gamma_{0}$ & $\delta$ \\
30 & 0.038 & 0.813 & 2.049 & 5.792 & 0.048 & 15.55 & 0.35 \\
60 & & & & & & & \\
\hline \hline
\end{tabular}

In the second approach, the attenuation in $\mathrm{dB} / \mathrm{m}$ was calculated using:

$$
\overline{A_{\text {ev }}}=A_{\text {ev }} / d
$$

where $A_{\text {ev }}$ was calculated using (10) with parameters from Table 9 at two different distances: $d=40 \mathrm{~m}$ (black curve in Fig. 7) and at $d=40 \lambda$ (red curve in Fig. 7), where $\lambda$ stands for the free-space wavelength at the operating frequency. The distance of $40 \mathrm{~m}$ in both first and the second approach was chosen as a distance far enough within the forest and longer than the wavelength at the lowest considered frequency. On the other hand, the distance of $40 \lambda$ was chosen as an electrically long path for the EM wave within the forest. The results are presented in Fig. 7, together with the measured data collected in [21].

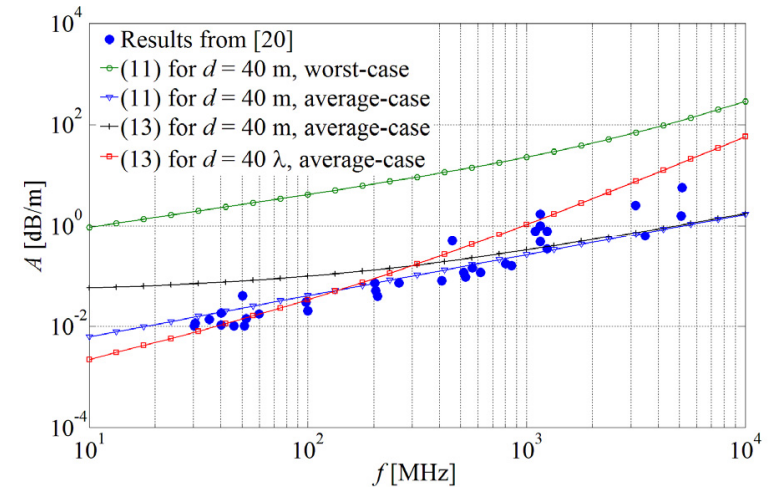

Fig. 7 Comparison to measured data from [21]

It is obvious from the presented results that different approaches for estimation of per-unit-length excess attenuation yield somewhat different results. However, all estimations fit within measured data within $1 \mathrm{~dB} / \mathrm{m}$, except at the highest frequencies presented in Fig. 7. Especially, the match between estimated per-unit-length of the excess attenuation and the measured data from [21] is below $0.5 \mathrm{~dB} / \mathrm{m}$, up to $1 \mathrm{GHz}$. Note that this range is significantly wider than the frequency range from which (10) was obtained. This comparison proves that the proposed analytical formulas can be used for the estimation of the excess attenuation of EM wave propagating within deciduous forests.

\section{CONCLUSION}

Excess attenuation of EM wave in the presence of deciduous forests is estimated using two novel analytical formulas. The parameters of the formulas were fitted to about 4,800 stochastic models that were numerically simulated and processed. It was shown that proposed analytical approach can be used to predict attenuation in deciduous forests up to at least $1 \mathrm{GHz}$.

\section{REFERENCES}

[1] A. Djordjević, Electromagnetics, School of Electrical Engineering, University of Belgrade, Serbia: Adacemic Mind 2012.

[2] S. Torrico, H. Bertoni, and R. Lang, "Modeling tree effects on path loss in a residential environment", IEEE Trans. on Antennas and Propagation, vol. 46, no. 6, pp. 872-880, June 1998.

[3] S. Seke, S. Ceran, O. Cerezci and A. Citkaya, "Attenuation of vegetation and snow on RF wireless communication", in $31^{\text {st }}$ International Review of Progress in Applied Computational Electromagnetics (ACES), 2015, pp 1-2.

[4] Y. Meng y, Y. Lee, and B. Ng, "Path loss modeling for near-ground VHF radio-wave propagation through forests with tree-canopy reflection effect", Progress In Electromagnetics Research $M$, Vol. $12,131-141,2010$

[5] Wang and K. Sarabandi, "A physics-based statistical model for wave propagation through foliage", IEEE Trans. on Antennas and Propagation, vol. 55, no. 3, pp. 958-968, March 2007.

[6] D. Olcan, B. Kolundzija, "Precise and efficient EM modeling of trees with WIPL-D code", in ACES Conference, 2004

[7] B. Mrdakovic, D. Olcan, B. Kolundzija, "Full-wave modeling of stochastic trees for radar cross section calculation", in $9^{\text {th }} E U$ Conf. on Antennas and Propagation (EuCAP), 2015, pp. 1-4.

[8] A. Tavakoli, K. Sarabandi, and F. Ulaby, "Horizontal propagation through Periodic vegetation canopies", IEEE Trans. on Antennas and Propagation, vol. 39, no. 7, pp. 1014-1023, July 1991.

[9] J. Azevedo and F. Santos, "An empirical propagation model for forest environments at tree trunk level", IEEE Trans. on Antennas and Propagation, vol. 59, no. 7, pp. 2357-2367, June 2011.

[10] R. Tewari, S. Swaurup and M. Roy,"Radio wave propagation through rain forests of India", IEEE Trans. on Antennas and Propagation, vol. 38, no. 4, pp. 1014-1023, April 1990.

[11] P. Horak and P. Pechac, "Frequency dependence of vegetation shadowing loss for satellite services", in $6^{h} E U$ Conf. on Antennas and Propagation (EuCAP), 2012, pp. 2445-2448.

[12] P. Chong, S. Yoo, S. Kim, and D. Kim, "Wind-blown foliage and human-induced fading in ground-surface narrowband communications at $400 \mathrm{MHz}$,, IEEE Trans. on Vehicular Technology, vol. 60, no. 4, pp. 1326-1336, May 2011.

[13] I. Rodriguez, R. Abreu, E. Almeida, M. Lauridsen, A. Loureiro and P. Mogensen, " $24 \mathrm{GHz}$ cmWave radio propagation through vegetation: suburban tree clutter attenuation", in $10^{\text {th }} E U$ Conf. on Antennas and Propagation (EuCAP), 2016, pp. 1-5.

[14] Attenuation in vegetation, ITU-R P.833-9, September 2016.

[15] J. Perovic, D. Olcan, "The Excess Attenuation of Electric Field in the Presence of Deciduous Trees", in $201624^{\text {th }}$ Telecommunications forum (TELFOR), pp. 6-14.

[16] MATLAB, The language of technical comp., MathWorks, 2011.

[17] Z. Michalewicz, D. Fogel, How to Solve It:Modern Heuristics, Appendix A, pp. 429-431. Springer 1999.

[18] J. Perovic, D. Olcan and B. Kolundzija, "The Excess Attenuation of Propagating Wave in the Presence of Human Crowds", in $11^{\text {th }} E U$ Conf. on Antennas and Prop. (EuCAP), 2017, pp 1335-1339.

[19] J. A. Nelder, R. Mead, "A Simplex method for function minimization”, The Computing Journal 7, pp. 308-313, 1965.

[20] B. Kolundzija, D. Olcan "Multiminima heuristic methods for antenna optimization", IEEE Trans. on Antennas and Propagation, vol. 54, no. 5, pp. 1405-1415, May 2006.

[21] A. A. Chukhlantsev, A. M. Shutko and S. P. Golovachev, "Attenuation of Electromagnetic Waves by Vegetation Canopies in the $100-10000 \mathrm{MHz}$ Frequency Band", Russian Academy of Sciences, Jurnal of Electronics, February 2003. Available: http://jre.cplire.ru/jre/feb03/4/text.html. 